\title{
Distributed moving horizon state estimation for sensor networks with low computation capabilities
}

\author{
Antonello Venturino*, $\dagger$, Cristina Stoica Maniu*, Sylvain Bertrand ${ }^{\dagger}$, Teodoro Alamo ${ }^{\ddagger}$ and Eduardo F. Camacho ${ }^{\ddagger}$ \\ *Université Paris-Saclay, \\ CNRS, CentraleSupélec \\ Laboratoire des signaux et systèmes \\ Gif-sur-Yvette, France, 91192 \\ E-mail: antonello.venturino@12s.centralesupelec.fr \\ cristina.stoica@12s.centralesupelec.fr
}

\begin{abstract}
This paper focuses on distributed state estimation for sensor network observing a discrete-time linear system. The provided solution is based on a Distributed Moving Horizon Estimation (DMHE) algorithm with consensus-based arrival cost and a pre-estimating Luenberger observer in the formulation of the local problem solved by each sensor. This leads to reduce the computation load, while preserving the accuracy of the estimation. Moreover, observability properties of local sensors are used for tuning the weights related to consensus information fusion built on an observability rank-based condition, in order to improve the convergence of the estimation error. Results obtained by Monte Carlo simulations are provided to compare the performance with existing approaches, in terms of accuracy of the estimations and computation time.
\end{abstract}

Index Terms-distributed state estimation, moving horizon estimation, Luenberger observer, sensor network, linear systems.

\section{INTRODUCTION}

In recent decades, the interest on distributed state estimation increased tremendously due to its variety of applications over sensor networks such as target tracking [1]-[2], exploration [3], monitoring [4], etc. The estimation algorithms used in these applications are mainly formulated as centralized sensor fusion architectures, where all the sensors transmit their local measurements to a central unit which processes the provided data to update the estimation [5]. In general, the centralized schemes are not scalable, since with the increasing number of sensors the complexity of the problem to solve also increases. Furthermore, the central unit cannot efficiently communicate with all sensors for large-scale sensor networks due to physical constraints (e.g. communication delay, limited communication bandwidth). Unlike centralized schemes [6], in distributed approaches [7]-[9] each sensor computes a local estimation using the information acquired only from locally connected neighbours. This can improve robustness to sensor failure exploiting redundancy [10] and also lower the communication burden since data is transmitted only among local nodes in the network.

The material in this paper was partially presented at the 24th International Conference on System Theory, Control and Computing, Sinaia, Romania, 2020 .
The continuous decreasing costs of sensors is making this application realizable, even tough there are still open problems to face with. In fact, distributed algorithms need to have particular properties in order to make them attractive for the industrial community. Thus, in the context of largescale systems, the algorithms must be scalable to be able to deal with large-scale networks, must have low computation load (necessary when dealing with low-cost sensors with low computation capabilities), must minimize the utilization of communication resources, etc. In [11], the authors reviewed several works about distributed state estimation over low-cost sensors networks, pointing out their characteristics, advantages, and challenging issues.

In recent years, Moving Horizon Estimation (MHE) techniques and its distributed counterpart (DMHE) have been used to successfully deal with large sensor networks [12]. The first idea of MHE proposed in [13] consists in estimating the current states by solving a least-square optimization problem penalizing on one hand the deviation between the measurements and the predicted outputs, and on the other hand the distance from the estimated state and the a priori information about the state. MHE is a practical strategy for constrained state estimation and a lot of research has been devoted to develop stability guarantees on the estimation error dynamics, e.g. [14]-[16]. Although this approach is functional for control engineers offering the freedom to tune the parameters of the cost function, a strength and a weakness of this approach is the use of an optimization problem. This problem has to be solved within the sampling period; however, for large-scale systems this issue becomes critical. There have been several attempts in trying to reduce the computation demanding of MHE. One idea is to add a pre-estimating observer in the formulation. The authors of [15] proposed a MHE strategy with a Luenberger observer that leads to good performance especially for large estimation horizons. They also provided an optimization problem to tune the parameters minimizing the effects of measurement noise and model errors. In [15], they generalized the formulation using a weight matrix for the penalty function and adding states constraints. A MHE with 
pre-estimation has been proposed also for non-linear systems [16]. The authors of [8] proposed a distributed algorithm for linear systems with a stability proof under weak observability conditions (exploiting a consensus-on-estimate and a consensus weight term in the DMHE formulation). Extension of this DMHE to a new formulation with pre-estimation has been investigated in [17]. However, the computation time issue becomes crucial, since usually the network is composed of low-cost sensors.

In this paper we extend the DMHE algorithm with preestimation presented in [17], based on the ideas of [8], [15]. A pre-estimating Luenberger observer is considered in the formulation of the local problem to be solved by each sensor, resulting in a significant reduction of the computation time. The main contribution of this paper covers:

- reducing the computation time required for solving the optimization problem;

- preserving the accuracy of the estimation errors, which allows the use of this type of algorithms for time-sensitive applications;

- better tuning the consensus weights associated to the network topology via a new observability rank-based weighted distributed algorithm which takes advantage of the local available information.

The paper is structured as follows. Section II presents the necessary theoretical background. Section III focuses on the proposed Distributed Moving Horizon Estimation approach. In Section IV a simulation example is proposed for performance illustration and analysis, while Section V reports some concluding remarks and future developments.

\section{NOTATIONS AND DEFINITIONS}

Consider the dynamics of the system described by the following discrete-time linear time invariant state-space equation:

$$
x_{t+1}=A x_{t}+w_{t},
$$

where $x_{t} \in \mathcal{X} \subseteq \mathbb{R}^{n}$ is the state and $w_{t} \in \mathcal{W} \subseteq \mathbb{R}^{n}$ is a zero mean white noise with covariance matrix $Q$. The sets $\mathcal{X}$ and $\mathcal{W}$ are assumed to be convex with the origin in their interior. The initial state $x_{0}$ is assumed to be unknown and modelled by a random variable of mean $\mu$ and covariance matrix $\Pi_{0}$. The measurements are performed by $M$ heterogeneous sensors:

$$
y_{t}^{i}=C^{i} x_{t}+v_{t}^{i}, \quad i=1, \ldots, M
$$

where $v_{t}^{i} \in \mathbb{R}^{p_{i}}$ is a zero mean white noise with covariance matrix $R^{i}$. Notice that, the superscript $(\cdot)^{i}$ indicates a variable that refers to the sensor $i$.

The sensor network is described by a digraph $\mathcal{G}=(\mathcal{N}, \mathcal{E})$, where the nodes $\mathcal{N}=\{1,2, \ldots, M\}$ represent the sensors and the edge $(j, i) \in \mathcal{E} \subseteq \mathcal{N} \times \mathcal{N}$ represents the communication link from sensor $j$ to sensor $i$. We assume that all the nodes have a self-loop $(i, i) \in \mathcal{E}, \forall i \in \mathcal{N}$. The neighbourhood $\mathcal{N}^{i}$ of the sensor $i$ is $\mathcal{N}^{i}=\{j \in \mathcal{N}:(j, i) \in \mathcal{E}\}$. We denote by $M^{i}=\operatorname{card}\left(\mathcal{N}^{i}\right)$ the number of nodes $j \in \mathcal{N}^{i}$.
Assuming that the topology of the sensor network is not time-varying, then a constant stochastic matrix $K \in \mathbb{R}^{M \times M}$ can be associated to the graph $\mathcal{G}$ such that the elements:

$$
\begin{aligned}
& k_{i j}>0 \text { if }(j, i) \in \mathcal{E}, \\
& k_{i j}=0 \text { otherwise, } \\
& \sum_{j=1}^{M} k_{i j}=1, \quad \forall i=1, \ldots, M .
\end{aligned}
$$

Section III-B will present a method that determines the value of each element $k_{i j}$, compatible with the graph $\mathcal{G}$, exploiting observability properties. The matrix $K$ will be used to compute the consensus terms in the DMHE algorithm described in Section III.

In order to recognize local, regional and collective information (as in [8]) we introduce a convenient notation. In particular, for a considered sensor $i$, an information is said local if it is related only to the node $i$. It is said regional concerning sensor $i$ if it is related to the nodes in $\mathcal{N}^{i}$. Finally, an information is said collective when the whole network is involved. Therefore, for the sake of clarity, we distinguish these information using different notations for local, regional and collective variables. Specifically, given a variable $z$, then $z^{i}, \bar{z}^{i}$ and $\mathbf{z}$ represent local, regional and collective data, respectively. For example, consider the sensor $i$ and its neighbourhood $\mathcal{N}^{i}=\left\{j_{1}, \ldots, j_{M^{i}}\right\}$, then its regional measurements are:

$$
\bar{y}_{t}^{i}=\bar{C}^{i} x_{t}+\bar{v}_{t}^{i},
$$

with the output vector $\bar{y}_{t}^{i}=\left[\left(y_{t}^{j_{1}}\right)^{\top} \ldots\left(y_{t}^{j_{M^{i}}}\right)^{\top}\right]^{\top} \in \mathbb{R}^{\bar{p}_{i}}$ of dimension $\bar{p}_{i}=\sum_{i \in \mathcal{N}^{i}} p_{i}$, the output matrix $\bar{C}^{i}=$ $\left[\left(C^{j_{1}}\right)^{\top} \ldots\left(C_{M^{i}}^{j^{\top}}\right)^{\top}\right]^{\top}$ and the measurement noise vector $\bar{v}_{t}^{i}=\left[\begin{array}{lll}\left(v_{t}^{j_{1}}\right)^{\top} & \ldots & \left(v_{t}^{j_{M^{i}}}\right)^{\top}\end{array}\right]^{\top}$. In addition, we denote by $\bar{R}^{i}$, the covariance matrix related to the regional noise vector $\bar{v}_{t}^{i}$ of sensor $i$, i.e., $\bar{R}^{i}=\operatorname{diag}\left(R^{j_{1}}, \ldots, R^{j_{M^{i}}}\right)$.

According to this terminology, three different observability notions can be defined.

Definition 1. The system is locally observable by sensor $i$ if the pair $\left(A, C^{i}\right)$ is observable. The system is regionally observable by sensor $i$ if the pair $\left(A, \bar{C}^{i}\right)$ is observable. The system is collective observable if the pair $(A, \mathbf{C})$ is observable. In the rest of the paper, by abuse of language, one will write that sensor $i$ is locally (respectively regionally) observable when the system is locally (respectively regionally) observable by sensor $i$.

\section{Proposed Distributed Moving Horizon Estimation With PRE-Estimation}

In this section, we extend the Distributed Moving Horizon Estimation with pre-estimation $\left(\mathrm{DMHE}_{\text {pre }}\right)$ presented in [17]. The proposed technique relies, as in the classical MHE paradigm [13], [14], on solving a constrained "least-square" optimization problem at each time $t$ that involves the initial state at time $t-N$, propagated forward via the dynamic model of the system (1), and the measurements performed by 
the considered sensors (2). A distributed setting also involves data shared by other sensors in the network [8]. The preestimation observer (introduced for centralized MHE by [15]) is used in the formulation of the proposed DMHE to help the mitigation of the effect of model uncertainty in the a priori estimation. Thus, the pre-estimation contributes to enhance the estimation accuracy. This strategy will also reduce the computation time required to solve the optimization problem, because the number of optimization variables involved is lower and does not depend on the horizon length $N$. Further details are given in the next sections.

\section{A. Local minimization problem}

In this section we formulate the proposed observability rank-based weighted DMHE technique with pre-estimation, hereafter denoted by $\mathrm{DMHE}_{w-p r e}$, in which each sensor $i \in \mathcal{N}$ solves its own local MHE problem based on regional measurements $\bar{y}_{t}^{i}$ and some shared information among the neighbours $j \in \mathcal{N}^{i}$. For a given estimation horizon length $N \geqslant 1$, each node $i \in \mathcal{N}$ at time $t$ determines the estimate $\hat{x}_{t \mid t}^{i}$ of the state $x_{t}$ by solving the following constrained minimization problem with pre-estimation:

$$
\begin{aligned}
\hat{x}_{t-N \mid t}^{i}=\arg \min _{\hat{x}_{t-N}^{i}} & J^{i}\left(t-N, t, \hat{x}_{t-N}^{i}, \hat{\bar{v}}^{i}, \Gamma_{t-N}^{i}\right) \\
\text { s.t. } \quad \hat{x}_{k+1}^{i} & =A \hat{x}_{k}^{i}+L^{i} \hat{\bar{v}}_{k}^{i}, \\
& \\
\hat{\bar{y}}_{k}^{i} & =\bar{C}^{i} \hat{x}_{k}^{i}+\hat{\bar{v}}_{k}^{i}, \\
\hat{x}_{k}^{i} & \in \mathcal{X}, \\
\forall k & =t-N, \ldots, t .
\end{aligned}
$$

The Luenberger gain $L^{i}$ is computed such that $\Phi^{i}=A-L^{i} \bar{C}^{i}$ is Schur stable when the sensor $i$ is regionally observable. Otherwise, as extrema ratio, $L^{i}$ can be computed in order to minimize the propagation of the error along the prediction horizon by keeping the spectrum radius of $\Phi^{i}$ the lowest possible.

A quadratic objective function $J^{i}$ is considered:

$$
J^{i}(\cdot)=\frac{1}{2} \sum_{k=t-N}^{t}\left\|\hat{\bar{v}}_{k}^{i}\right\|_{\left(\bar{R}^{i}\right)^{-1}}^{2}+\Gamma_{t-N}^{i}\left(\hat{x}_{t-N}^{i}, \hat{\bar{x}}_{t-N \mid t-1}^{i}\right),
$$

where the initial penalty function $\Gamma_{t-N}^{i}\left(\hat{x}_{t-N}^{i}, \hat{\bar{x}}_{t-N \mid t-1}^{i}\right)$ in (9) defined as follows:

$$
\Gamma_{t-N}^{i}(\cdot)=\frac{1}{2}\left\|\hat{x}_{t-N}^{i}-\hat{\bar{x}}_{t-N \mid t-1}^{i}\right\|_{\left(\bar{\Pi}_{t-N \mid t-1}^{i}\right)^{-1}}^{2},
$$

involves two consensus terms described below.

We denote by $\hat{\bar{x}}_{t-N \mid t-1}^{i}$ the weighted average state estimation computed by the neighbourhood $\mathcal{N}^{i}$ as follows:

$$
\hat{\bar{x}}_{t-N \mid t-1}^{i}=\sum_{j \in \mathcal{N}^{i}} k_{i j} \hat{x}_{t-N \mid t-1}^{j},
$$

where $\hat{x}_{t-N \mid t-1}^{j}$ is the second estimated state in the sequence computed at the previous time by sensor $j$. Notice that the penalty function $\Gamma_{t-N}^{i}$ includes a consensus-on-estimates term, in the sense that it penalizes deviations of $\hat{x}_{t-N}^{i}$ from $\hat{\bar{x}}_{t-N \mid t-1}^{i}$. It helps to improve the accuracy of the local estimates and it is necessary to guarantee convergence of the state estimates to the state of the observed system even if it lacks of regional observability [8].

The positive definite matrix $\bar{\Pi}_{t-N \mid t-1}^{i}$ is computed as in [8]. For the sake of completeness, we recall here the procedure to compute it by:

$$
\bar{\Pi}_{t-N \mid t-1}^{i}=\sum_{j \in \mathcal{N}^{i}} M^{j} k_{i j}^{2} \Pi_{t-N \mid t-1}^{j},
$$

where the update of $\Pi_{t-N \mid t-1}^{i}$ is performed by the sensor $i$ on the basis of regionally available information. In particular, the matrix $\Pi_{t-N \mid t-1}^{i}$, with $i \in \mathcal{N}$, is given by one iteration of the difference Riccati equation associated to a Kalman filter for the system:

$$
\left\{\begin{array}{l}
x_{t-N}=A x_{t-N-1}+w_{t-N-1} \\
\bar{z}_{t-N}^{i}=\overline{\mathcal{O}}_{N}^{i} x_{t-N}+\bar{V}_{t-N}^{i}
\end{array}\right.
$$

where $\bar{V}_{t-N}^{i}$ represents the measurements noise and $\overline{\mathcal{O}}_{N}^{i}$ defines the $i$-th sensor regional observability matrix:

$$
\overline{\mathcal{O}}_{N}^{i}=\left[\begin{array}{llll}
\left(\bar{C}^{i}\right)^{\top} & \left(\bar{C}^{i} A\right)^{\top} & \cdots & \left(\bar{C}^{i} A^{N-1}\right)^{\top}
\end{array}\right]^{\top} .
$$

Then defining:

$$
\begin{aligned}
& \mathcal{C}_{N}^{i}=\left[\begin{array}{cccc}
0 & 0 & \cdots & 0 \\
\bar{C}^{i} & 0 & \cdots & 0 \\
\vdots & \vdots & \ddots & \vdots \\
\bar{C}^{i} A^{N-2} & \bar{C}^{i} A^{N-3} & \cdots & \bar{C}^{i}
\end{array}\right] \in \mathbb{R}^{\bar{p}_{i} N \times n(N-1)}, \\
& \bar{R}_{N}^{i}=\operatorname{diag}\left(\bar{R}^{i}, \ldots, \bar{R}^{i}\right) \in \mathbb{R}^{\bar{p}_{i} N \times \bar{p}_{i} N} \\
& Q_{N-1}=\operatorname{diag}(Q, \ldots, Q) \in \mathbb{R}^{n(N-1) \times n(N-1)} \\
& \operatorname{Cov}\left[\bar{V}_{t}^{i}\right]=\bar{R}_{N}^{* i}=\bar{R}_{N}^{i}+\mathcal{C}_{N}^{i} Q_{N-1}\left(\mathcal{C}_{N}^{i}\right)^{\top}
\end{aligned}
$$

and setting the covariance of the estimate $\hat{x}_{t-N-1}^{i}$ as:

$$
\Pi_{t-N-1 \mid t-2}^{* i}=\left(\left(\bar{\Pi}_{t-N-1 \mid t-2}^{i}\right)^{-1}+\left(\bar{C}^{i}\right)^{\top}\left(\bar{R}^{i}\right)^{-1} \bar{C}^{i}\right)^{-1}
$$

the resulting Riccati recursive equation is given by:

$$
\begin{aligned}
\Pi_{t-N \mid t-1}^{i}= & A \Pi_{t-N-1 \mid t-2}^{* i} A^{\top}+Q-A \Pi_{t-N-1 \mid t-2}^{* i}\left(\overline{\mathcal{O}}_{N}^{i}\right)^{\top} \\
& \cdot\left(\overline{\mathcal{O}}_{N}^{i} \Pi_{t-N-1 \mid t-2}^{* i}\left(\overline{\mathcal{O}}_{N}^{i}\right)^{\top}+\bar{R}_{N}^{* i}\right)^{-1} \\
& \cdot \overline{\mathcal{O}}_{N}^{i} \Pi_{t-N-1 \mid t-2}^{* i} A^{\top} .
\end{aligned}
$$

Since the communication network topology is assumed to be time-invariant, these equations could be computed off-line. However, once the matrices $\Pi_{t-N \mid t-1}^{i}$ have been computed, we perform a consensus weights update in order to compute the matrices $\bar{\Pi}_{t-N \mid t-1}^{i}$ according to (12).

Remark 1. In the proposed DMHE formulation the sequence of the input noise $\left\{w_{k}\right\}_{k=t-N}^{t-1}$ are no longer considered as optimization parameters, contrary to DMHE of [8]. This allows to reduce the computation time required to solve the optimization problem. 


\section{B. Weights tuning}

In [8], the authors proposed an algorithm to compute $K$, according to (3) and compatible with $\mathcal{G}=(\mathcal{N}, \mathcal{E})$, to ensure the stability of their DMHE. This algorithm requires knowledge about the global network topology and, thus, the consensus weights have to be globally recomputed when the network topology changes. In order to overcome this issue, we further present a new observability rank-based weighted approach built on only local information available to each sensors $i \in$ $\mathcal{N}$, thus representing an enhancement w.r.t. the algorithm in [8]. The proposed technique relies on observability properties associated to each sensor. With the intention to enhance the convergence of the consensus terms, the proposed method gives major importance to sensors that have better regional observability properties. A description of this approach is presented below.

Observability rank-based weights technique. Consider a sensor $i$. Its regional observability matrix:

$$
\overline{\mathcal{O}}_{n}^{i}=\left[\begin{array}{llll}
\left(\bar{C}^{i}\right)^{\top} & \left(\bar{C}^{i} A\right)^{\top} & \cdots & \left(\bar{C}^{i} A^{n-1}\right)^{\top}
\end{array}\right]^{\top}
$$

is of full rank if and only if the the pair $\left(A, \bar{C}^{i}\right)$ is completely observable, i.e. $\operatorname{rank}\left(\overline{\mathcal{O}}_{n}^{i}\right)=n$. Otherwise, its rank is less than $n$. For the sake of simplicity, we denote by $\rho_{\mathcal{O}}^{i}=\operatorname{rank}\left(\overline{\mathcal{O}}_{n}^{i}\right)$. This information could be used as reliability of sensor $i$ when choosing the weights, which according to (3) must be averaged among the neighbours, resulting in:

$$
k_{i j}=\frac{\rho_{\mathcal{O}}^{j}}{\sum_{j \in \mathcal{N}^{i}} \rho_{\mathcal{O}}^{j}}, \quad \forall j \in \mathcal{N}^{i} .
$$

It is worth noticing that each row of the matrix $K$ concerns one sensor, e.g., the $i$-th row can be computed by sensor $i$ using data coming from its neighbours $j \in \mathcal{N}^{i}$ solely. Hence, it can easily be recomputed online if the topology of the network changes.

\section{Network information exchange}

It is worth highlighting the way each node exchanges the information with its neighbours. To this end, we list here some assumptions that play a major role:

- the network could be composed by heterogeneous sensors;

- the sensors are time synchronized.

This implies that the matrices $C^{i}$ in (2) can be different for all $i \in \mathcal{N}$. Furthermore, if we assume also that the network topology is time-invariant, then the neighbourhood $\mathcal{N}^{i}$ is known a priori and it is not necessary to exchange the information of the matrices $C^{i}$ and $R^{i}$ at each time. Moreover, this allows one to compute off-line the Luenberger gains $L^{i}$.

\section{DMHE procedure}

Finally, the procedure of the proposed distributed scheme is described in Algorithm 1.

Notice that the steps 10,16 and 19 in the procedure regarding exchanging information could be rearranged to have just one synchronization. However, for clarity reasons with respect to calculation details, they have been described this way.

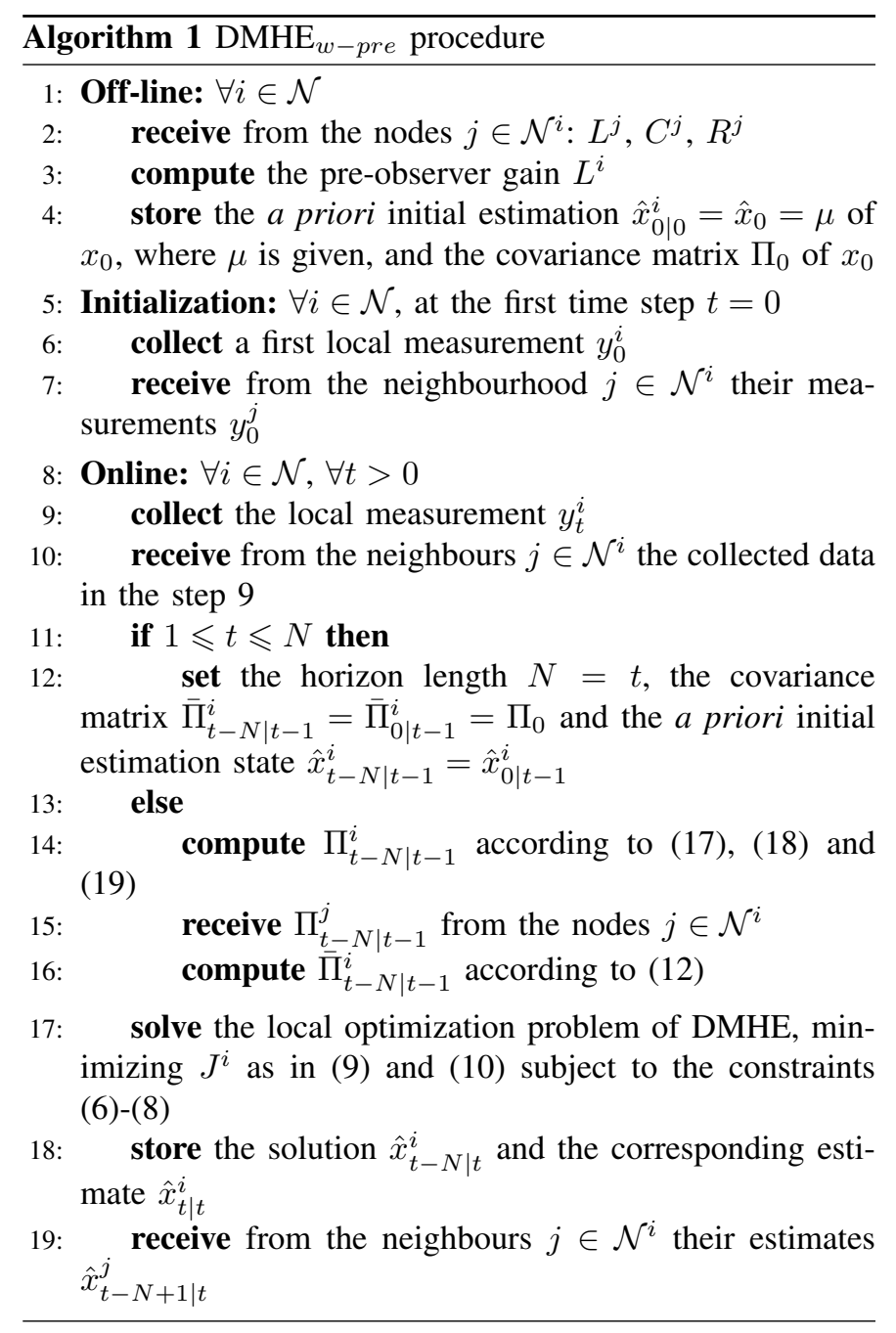

\section{Simulation RESUlts}

In this section, the effectiveness of the proposed DMHE algorithm with pre-estimation is investigated. In order to evaluate its performance, the proposed technique is compared to the centralized MHE of [14] as well as the DMHE algorithms of [8] and [17]. To this end, we consider the system in [8], recalled below:

$$
x_{t+1}=\left[\begin{array}{cccc}
0.9962 & 0.1949 & 0 & 0 \\
-0.1949 & 0.3819 & 0 & 0 \\
0 & 0 & 0 & 1 \\
0 & 0 & -1.21 & 1.98
\end{array}\right] x_{t}+w_{t},
$$

where $x_{t}=\left[\begin{array}{llll}x_{1, t} & x_{2, t} & x_{3, t} & x_{4, t}\end{array}\right]^{\top}$ is the state vector and $w_{t} \in \mathbb{R}^{4}$ is a zero-mean white noise with covariance matrix $Q=\operatorname{diag}(0.0012,0.038,0.0012,0.038)$. Notice that the system is unstable since the eigenvalues of $A$ are 0.9264 , $0.4517,0.99 \pm 0.4795 i$ and $|0.99 \pm 0.4795 i|>1$. For this example, one considers $\mathcal{X}=\mathbb{R}^{4}$.

The initial values of the algorithms have been set as $\mu=$ $\left[\begin{array}{llll}0 & 0 & 0 & 0\end{array}\right]^{\top}, \Pi_{0}=I_{4}$. 
To compare the results of all algorithms we use $M=9$ sensors for the distributed algorithms. For the centralized scheme we consider the following measurement equation:

$$
y_{t}=\left[\begin{array}{llll}
1 & 0 & 0 & 0 \\
0 & 0 & 1 & 0
\end{array}\right] x_{t}+v_{t},
$$

with $\operatorname{Var}\left(v_{t}\right)=R=I_{2}$. The considered distributed approaches are using the following measurement equations:

$$
\begin{aligned}
& y_{t}^{i}=\left[\begin{array}{llll}
1 & 0 & 0 & 0
\end{array}\right] x_{t}+v_{t}^{i} \quad \text { if } i=1,2,6 \\
& y_{t}^{i}=\left[\begin{array}{llllll}
0 & 0 & 1 & 0 & x_{t}+v_{t}^{i} & \text { if } i=3,4,9
\end{array}\right. \\
& y_{t}^{i}=\left[\begin{array}{llll}
0 & 0 & 0 & 0
\end{array}\right] x_{t}+v_{t}^{i} \quad \text { if } i=5,7,8
\end{aligned}
$$

where $\operatorname{Var}\left(v_{t}^{i}\right)=R^{i}=1, i=1, \ldots, 9$. The nodes are connected as reported by the graph in Fig. 1 and the transition matrix $K$ is computed with the observability rankbased weighted method (21). The different colors highlight the regional observability properties of each sensor $i$, thus of the pair $\left(A, \bar{C}^{i}\right)$. In particular, the green nodes mean that the pair $\left(A, \bar{C}^{i}\right)$ is completely observable, the yellow nodes are at least detectable, the orange nodes are not detectable and the red node has no sensing information, i.e. $\rho_{\mathcal{O}}^{i}=0$.

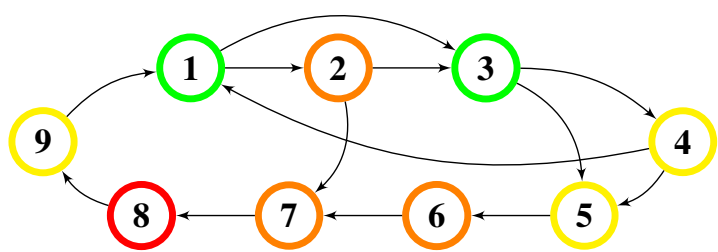

Fig. 1: Sensor network.

To evaluate the performance of the proposed algorithm we take into account two performance metrics both averaged over the nodes in the network. The first metric is the Root Mean Square Error (RMSE) computed as follows:

$$
\operatorname{RMSE}_{t}=\frac{1}{M} \sum_{i=1}^{M}\left\|x_{t}-\hat{x}_{t \mid t}^{i}\right\|,
$$

where $M=9$ for distributed schemes and $M=1$ for the centralized one. The second metric is the computation time $\tau$ needed by each algorithm.

We consider two simulations cases with time duration $t_{f}=$ $50 s$.

Case 1. Setting the horizon length $N=4$, one hundred Monte Carlo trials have been performed with each component of the initial state $x_{0}$ uniformly distributed in $[-100,100]$.

In Fig. 2 it is noticeable that the proposed algorithm DMHE $_{w-p r e}$ is able to converge and also shows better performance vs. the DMHE of [8] and [17], which are one above the other because these last two have very similar RMSEs. This is more evident in Fig. 3 which shows the sum of RMSEs in the transient period and in the steady-state.

Regarding the second performance metric in Fig. 4, it is clear that the algorithms with pre-estimation are less time demanding than the DMHE of [8] and the centralized MHE. In fact, the computation time $\tau$ is reduced in terms of percentage circa $27 \%$ w.r.t. the DMHE of [8] and $49 \%$ w.r.t. the

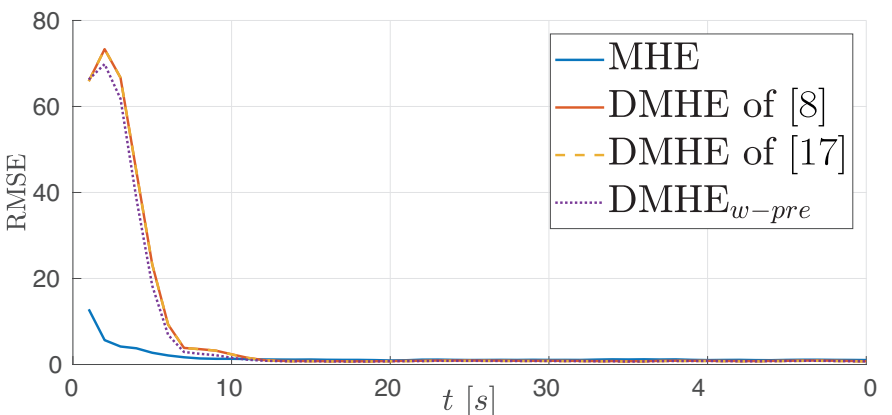

Fig. 2: Time behaviour of the trials-averaged RMSE for 100 Monte Carlo trials.

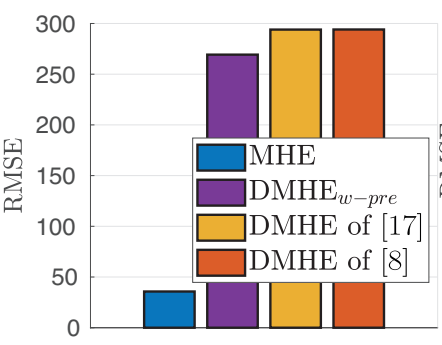

(a) Transient period

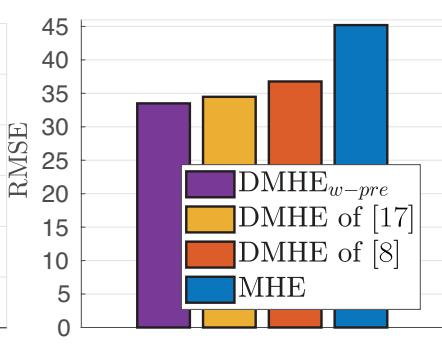

(b) Steady-state
Fig. 3: Sum of RMSEs in the transient period (for $t \in$ $[1, \ldots, 9] s$ ) and in the steady-state (for $t \in[10, \ldots, 50] s$ ).

centralized MHE. Notice that the computation time of DMHE in [17] and the extended version proposed in this paper are the same due to the fact that the difference of the extended version (DMHE ${ }_{w-p r e}$ ) concerns only consensus weights computation.

Case 2. To better evaluate the proposed algorithm, a second simulation has been performed with the same parameters as the first case except two crucial variables. First of all, a different horizon length is used, i.e. $N \in\{2,3, \ldots, 10\}$. The matrix $K$ used in this simulation results in a convex combination with the parameter $\varepsilon \in\{0,0.1, \ldots, 1\}$ of the matrices $\tilde{K}$ and $\hat{K}$ :

$$
K=\varepsilon \tilde{K}+(1-\varepsilon) \hat{K}
$$

where $\tilde{K}$ is computed with the proposed observability rankbased weighted method, while $\hat{K}$ is computed as in [8]. Moreover, a set of 100 different initial states $\left\{x_{0, z}\right\}_{z=0}^{100}$ has been uniformly generated from $[-100,100]$. Therefore, this

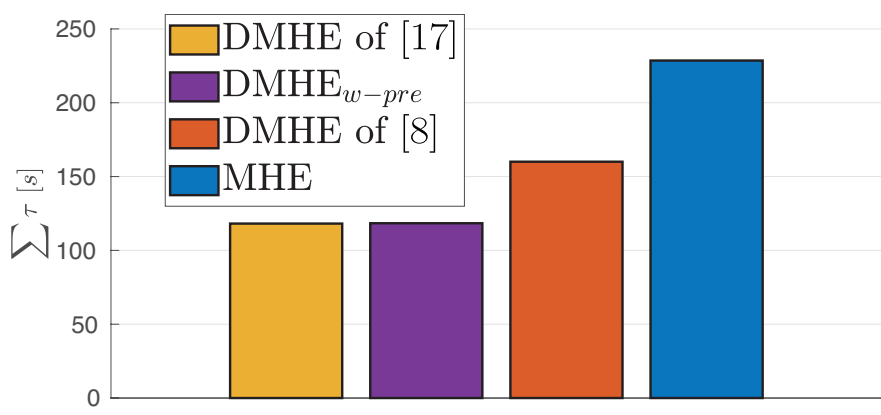

Fig. 4: Sum of the trials-averaged computation times $\tau$ for 100 Monte Carlo trials. 
case simulation is composed by 9900 trials as result of the Cartesian product $N \times \varepsilon \times\left\{x_{0, z}\right\}$.

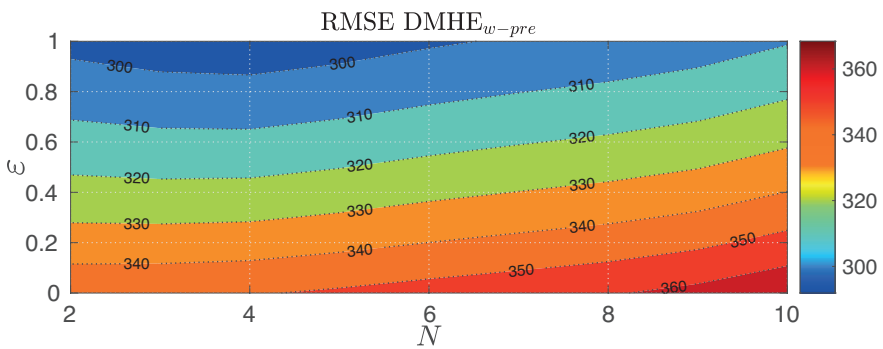

Fig. 5: Sum of $x_{0}$-averaged RMSEs of the proposed DMHE $_{w-p r e}$ with $K$ varying as in (23).

Figure 5 shows the RMSEs of the $\mathrm{DMHE}_{w-\text { pre }}$ with $K$ as in (23). The RMSE is averaged among the $z=1, \ldots, 100$ trials by changing $x_{0, z}$, computed as follows:

$$
\operatorname{RMSE}_{(N, \varepsilon)}=\frac{1}{100} \sum_{z=1}^{100} \sum_{t=1}^{t_{f}} \operatorname{RMSE}_{t, x_{0, z}},
$$

which results in a function of the horizon length $N$ and the parameter $\varepsilon$. As we can see, along the $N$-axis the slope of the level curves of the RMSE changes mildly, with a minimum for $N=\{3,4\}$. Along the $\varepsilon$-axis it is evident that the minimum is at $\varepsilon=1$, thus when $K=\tilde{K}$. This means that Fig. 5 shows that the performance in terms of RMSE of the proposed observability rank-based weighted method is better than Algorithm 1 (then $\hat{K}$ ) in [8].

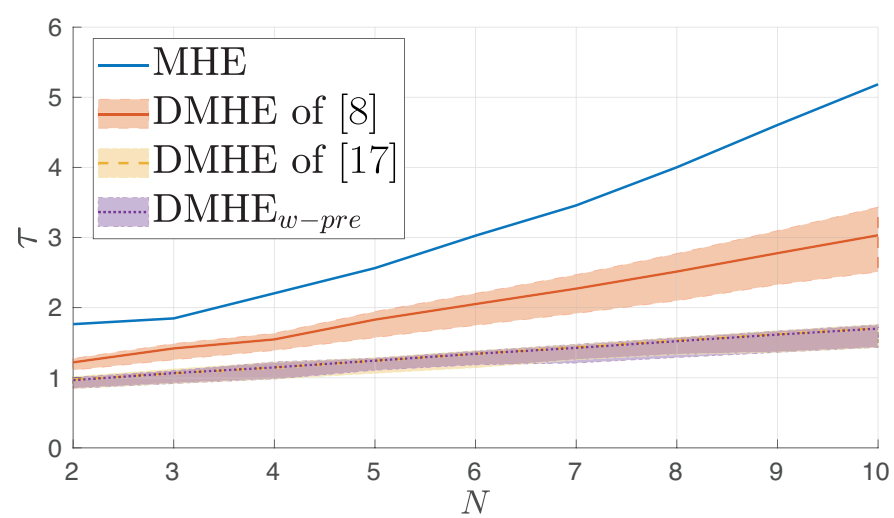

Fig. 6: $x_{0}-\varepsilon$-averaged computation time $\tau$.

Idem for this case simulation, the computation time is lower for the two DHMEs with pre-estimation, thus also for the proposed observability rank-based weighted method, as shown in Fig. 6. This figure shows the computation time $\tau$ averaged over the trials in which $\varepsilon$ and $x_{0}$ change, that results in $\tau$ as function of $N$. The slope of this function is the same for the two DMHEs with pre-estimation, and is lower than the DMHE in [8] and the centralized MHE. Moreover, Fig. 6 also shows the limits representing the minimum and maximum computation time of the DMHE algorithms. It can be noticed that these limits are narrower and less deviating w.r.t. $N$ for the algorithms with pre-estimation. This clearly shows the very promising performance of the proposed algorithm for implementation on networks of sensors with low computation capabilities.

\section{CONCLUSiON}

In this paper, a distributed architecture based on Moving Horizon Estimation (MHE) paradigm is proposed for state estimation of discrete-time linear systems by sensor networks. The main merits of the proposed approach can be summarized as follows: 1) the computation time is considerably reduced by means of the pre-estimation observer; 2) the accuracy of the estimation errors is improved both in the transient period and in the steady-state w.r.t. the one of the original formulation [8], as result of choosing the consensus weight matrix $K$ with the presented rank-based weighted method; 3) moreover, this method allows each sensor to determine its consensus weights on the basis of only local provided information contrary to Algorithm 1 in [8] that, instead, needs knowledge about the global network topology. Thus it is suitable for a fully distributed scheme and could be extended for time-varying topology.

Simulation results have shown the effectiveness of the proposed DMHE algorithm even in presence of weak regional observability conditions induced by some sensors of the considered network.

Current work focuses on implementation of the proposed algorithm on a real multi-robot system for distributed localization.

\section{ACKNOWLEDGMENT}

This work was supported by the Agencia Estatal de Investigación (AEI)-Spain under Grant PID2019-106212RBC41/AEI/10.13039/501100011033 and the European Research Council under the advanced grant OCONTSOLAR Grant agreement ID: 789051.

The first author acknowledges the support of the STIC Doctoral School (Paris-Saclay University) for a 3-month visit at University of Seville.

\section{REFERENCES}

[1] A. Albert and L. Imsland, "Survey: mobile sensor networks for target searching and tracking," Cyber-Physical Systems, vol. 4, no. 2, pp. 5798, 2018.

[2] A. Petitti, D. Di Paola, A. Rizzo, and G. Cicirelli, "Consensus-based distributed estimation for target tracking in heterogeneous sensor networks," in 50th IEEE Conference on Decision and Control and European Control Conference, 2011, pp. 6648-6653.

[3] D. Fox, J. Ko, K. Konolige, B. Limketkai, D. Schulz, and B. Stewart, "Distributed multirobot exploration and mapping," Proceedings of the IEEE, vol. 94, no. 7, pp. 1325-1339, 2006.

[4] N. Forti, G. Battistelli, L. Chisci, S. Li, B. Wang, and B. Sinopoli, "Distributed joint attack detection and secure state estimation," IEEE Transactions on Signal and Information Processing over Networks, vol. 4, no. 1, pp. 96-110, 2017.

[5] F. Tedesco, C. Ocampo-Martinez, A. Casavola, and V. Puig, "Centralized and distributed command governor approaches for water supply systems management," IEEE Transactions on Systems, Man, and Cybernetics: Systems, vol. 48, no. 4, pp. 586-595, 2016.

[6] D. Merhy, T. Alamo, C. Stoica Maniu, and E. F. Camacho, "Zonotopic constrained Kalman filter based on a dual formulation," in IEEE Conference on Decision and Control, 2018, pp. 6396-6401.

[7] F. F. Rego, A. M. Pascoal, A. P. Aguiar, and C. N. Jones, "Distributed state estimation for discrete-time linear time invariant systems: A survey," Annual Reviews in Control, vol. 48, pp. 36-56, 2019. 
[8] M. Farina, G. Ferrari-Trecate, and R. Scattolini, "Distributed moving horizon estimation for linear constrained systems," IEEE Transactions on Automatic Control, vol. 55, no. 11, pp. 2462-2475, 2010.

[9] M. Farina, G. Ferrari-Trecate, and R. Scattolini, "Distributed moving horizon estimation for nonlinear constrained systems," International Journal of Robust and Nonlinear Control, vol. 22, no. 2, pp. 123-143, 2012.

[10] T. Tošić, N. Thomos, and P. Frossard, "Distributed sensor failure detection in sensor networks," Signal Processing, vol. 93, no. 2, pp. 399-410, 2013.

[11] S. He, H.-S. Shin, S. Xu, and A. Tsourdos, "Distributed estimation over a low-cost sensor network: A review of state-of-the-art," Information Fusion, vol. 54, pp. 21-43, 2020.

[12] G. Battistelli, "Distributed moving-horizon estimation with arrival-cost consensus," IEEE Transactions on Automatic Control, vol. 64, no. 8, pp. 3316-3323, 2018.

[13] K. R. Muske, J. B. Rawlings, and J. H. Lee, "Receding horizon recursive state estimation," in American Control Conference, 1993, pp. 900-904.

[14] C. V. Rao, J. B. Rawlings, and J. H. Lee, "Constrained linear state estimation - a moving horizon approach," Automatica, vol. 37, no. 10, pp. 1619-1628, 2001.

[15] D. Sui and T. A. Johansen, "Linear constrained moving horizon estimator with pre-estimating observer," Systems \& Control Letters, vol. 67, pp. 40-45, 2014.

[16] R. Suwantong, S. Bertrand, D. Dumur, and D. Beauvois, "Stability of a nonlinear moving horizon estimator with pre-estimation," in American Control Conference, 2014, pp. 5688-5693.

[17] A. Venturino, S. Bertrand, C. Stoica Maniu, T. Alamo, and E. F. Camacho, "Distributed moving horizon estimation with pre-estimating observer," in 24th International Conference on System Theory, Control and Computing, 2020, pp. 174-179. 